\title{
COMPETÊNCIAS DOS PROFISSIONAIS DE SAÚDE NO REFERENCIAL DAS FUNÇÕES ESSENCIAIS DE SAÚDE PÚBLICA: contribuição para a construção de Projetos Pedagógicos na Enfermagem
}

Regina Rigatto Witt* Maria Cecília Puntel de Almeida**

\section{Resumo}

A construção de competências dos profissionais de saúde no referencial das Funções Essenciais de Saúde Pública (FESP) faz parte de um conjunto de iniciativas que buscam dar resposta às necessidades dos serviços para a resolução dos problemas de saúde, considerando-se as transformações ocorridas no setor saúde e no mundo do trabalho. O objetivo deste trabalho é fazer uma reflexão teórica sobre a temática de competências profissionais, a fim de contribuirmos para a construção de projetos político-pedagógicos para o ensino da saúde e da enfermagem em especial. Para isto, apresentamos o modelo de competências, essas, no referencial das FESP e as propostas apresentadas até então, tendo em vista a construção de modelos de formação interdisciplinares, críticos e reflexivos.

Descritores: competência profissional; enfermagem em saúde pública; educação em enfermagem

\begin{abstract}
The development of competencies among health professionals in relation to the Essential Public Health Functions (EPHF) is part of a number of initiatives that are meant to face the need for services that can lead to the resolution of health problems, considering the transformations occurred in the health sector and in the workplace. This article's objective is to make a theorical reflection on the theme of professional competencies, so that we can contribute to the development of politicalpedagogical projects for health training and primarily for nursing training. For that matter, we introduce the competency-based model. These competencies are in accordance with the EPHF and with the competencies proposed so far, and are aimed at contributing to the development of interdisciplinary, critical, and reflexive education models.

Descriptors: professional competence; public health nursing; nursing education

Title: Health Professionals competencies in relation to Essential Public Health Functions: contribution to the design of Pedagogical Projects in Nursing
\end{abstract}

\section{Resumen}

La construcción de las competencias de los profesionales de salud en el marco de las Funciones Esenciales de Salud Pública (FESP) es parte de un conjunto de iniciativas que buscan dar respuesta a las necesidades de los servicios para resolver los problemas de salud, considerando las transformaciones ocurridas en el sector de la salud y en el mundo del trabajo.El objetivo es hacer una reflexión teorica sobre el tema de las competencias profisionales a fin de contribuir para la construcción de proyectos políticopedagógicos en la enseñanza de la salud y de la Enfermería en especial. Para ello, se presenta el modelo de competencias en el marco de las FESP y teniendo en cuenta los modelos de formación interdisciplinares, críticos y reflexivos.

Descriptores: competencia profesional; enfermeria en salud pública; educación en enfermería

Título: Competencias de los profesionales de salud en el marco de las funciones esenciales de salud pública: contribución para la construcción de proyectos pedagógicos en enfermería

\section{Introdução}

A construção de competências dos profissionais de saúde, no referencial das Funções Essenciais de Saúde Pública (FESP), faz parte de um conjunto de iniciativas que buscam dar resposta às necessidades dos serviços, para a resolução dos problemas de saúde, tendo em vista as transformações estruturais ocorridas no setor saúde e no mundo do trabalho devido, sobretudo, aos processos de globalização e integração econômica e às mudanças nos paradigmas em saúde pública segundo a Organização Pan-Americana da Saúde - OPAS ${ }^{(1)}$.

Em diversos países, os processos de reforma desencadeados nos anos 90 são conformados pelas demandas originadas da evolução demográfica e epidemiológica, do desenvolvimento tecnológico e do surgimento de novos atores ${ }^{(2)}$ No Brasil, este período é marcado pelo avanço do processo de descentralização do setor saúde, que converge para a ampliação da capacidade de governo local, associada à melhor estruturação da atenção básica ${ }^{(3)}$. O trabalho, neste nível, baseia-se em uma nova concepção de saúde, não mais centrada, somente, na assistência à doença mas, sobretudo, na promoção da qualidade de vida e intervenção nos fatores que a colocam em risco - pela incorporação das ações programáticas de uma forma mais abrangente e do desenvolvimento de ações intersetoriais, de acordo com a proposição do Sistema Único de Saúde - SUS ${ }^{(4,5)}$.
Estas transformações repercutiram no modelo de formação dos profissionais da saúde, culminando com a construção coletiva das Diretrizes Curriculares Nacionais para os Cursos Universitários da Área da Saúde, na busca de um perfil profissional com competências, habilidades e conteúdos contemporâneos para atuarem no Sistema Único de Saúde, considerando o processo de Reforma Sanitária brasileira(6)

Este processo ocorreu em meio a uma articulação entre as instituições de formação e o sistema de saúde. A estreita inter-relação entre educação e prática, o posicionamento adequado - com respeito às competências críticas das instituições e da força de trabalho em saúde pública - são considerados pela OPAS ${ }^{(7)}$ componentes iniludíveis da planificação e gestão estratégica institucional. Porém, é preciso compreender o contexto e as repercussões da noção de competência, enquanto categoria ordenadora da relação trabalho-educação, a fim de que possamos discutir as limitações e as possibilidades de sua utilização na reorganização do trabalho em saúde pública e nos processos de formação em enfermagem, a fim de contribuirmos para a construção de projetos políticopedagógicos.

Portanto, este trabalho tem como objetivo fazer uma reflexão teórica sobre a temática de competências profissionais, a fim de ampliar o debate e contribuir para a construção de projetos político-pedagógicos para o ensino da saúde e da enfermagem em especial.

\footnotetext{
* Enfermeira, Mestre em Saúde Pública, Professora Assistente da Escola de Enfermagem da Universidade Federal do Rio Grande do Sul.

** Enfermeira, Livre-Docente em Enfermagem, Professora Titular da Escola de Enfermagem de Ribeirão Preto da Universidade de São Paulo. E-mail dos autores: witt@orion.ufrgs.br e cecília@eerp.usp.br
} 


\section{O modelo de competências e suas repercussões no trabalho e na educação}

O modelo de competências foi primeiramente incorporado pelo mundo do trabalho e inserido, posteriormente, nas propostas de formação e atualização profissionais. As alterações produzidas por este modelo, resultam em deslocamento de noções: dos saberes à competência, na esfera educativa; da qualificação à competência, na esfera do trabalho(8). Os objetivos da adoção do modelo das competências são adequar a formação da força de trabalho às novas exigências do sistema produtivo, possibilitando flexibilização do mercado de trabalho e unificando o sistema de qualificação profissional(9).

A competência, como uma categoria de análise, ou seja, "aquela que retém historicamente as relações sociais fundamentais e podem ser consideradas balizas para o conhecimento do objeto nos seus aspectos gerais"(10:94), condensa elementos significativos do desenvolvimento capitalista ocorrido nas últimas décadas com mudanças tecnológicas e de organização que configuram o mundo produtivo, a partir de algumas características tendenciais: flexibilização da produção e reestruturação das ocupações; integração de setores da produção; multifuncionalidade e polivalência dos trabalhadores; valorização dos saberes dos trabalhadores não ligada ao trabalho prescrito ou conhecimento formalizado(11)

Neste contexto, o trabalho passa a ser compreendido como uma série de eventos (situações singulares que se entrechocam), relacionando-se uns com os outros, num regime de modificação (e não de repetição) da maneira de produzir. Estes eventos devem ser analisados, critica e sistematicamente, quando possível, e antecipados preventivamente. O processo de trabalho deixa de ser compreendido como uma seqüência de fatos previsíveis para ser considerado uma seqüência de eventos ${ }^{(12)}$.

A emergência da noção de competência atende, pelo menos a três propósitos ${ }^{(12)}$ :

Reordenar conceitualmente a compreensão da relação trabalho-educação, desviando o foco dos empregos, das ocupações e das tarefas para o trabalhador em suas implicações subjetivas com o trabalho;

Institucionalizar novas formas de educar/formar os trabalhadores e de gerir o trabalho internamente às organizações e no mercado de trabalho em geral, sob novos códigos profissionais em que figuram as relações contratuais, de carreira e de salários;

Formular padrões de identificação da capacidade real do trabalhador para determinada ocupação, de tal modo que possa haver mobilidade entre as diversas estruturas de emprego em nível nacional e, também, em nível regional.

O diploma deixa de ser o único ou principal pressuposto para o emprego e passa a concorrer com as formações ditas qualificantes que visam a adaptação ao emprego. A certeza do emprego, que era motivada pela crença na relação linear escolaridade-formação-emprego, se transforma numa aposta incerta, em que as perspectivas de emprego ou auto-emprego, dependem, exclusivamente, de atributos individuais. A racionalização do trabalho solicita um novo tipo de trabalhador, capaz de compreender e participar de um ambiente onde as decisões são mais complexas e as interações sociais mais numerosas.

As noções de competência e empregabilidade têm sido relacionadas como forma de educar/formar os trabalhadores e de gerir o trabalho internamente às organizações e no mercado de trabalho em geral(12). A distinção dessas noções se dá no sentido de que a primeira se realiza internamente à empresa, enquanto a segunda se realiza externamente à empresa. A competência se associaria a uma postura de gestão adequada à organização qualificante, que reúne atributos como autonomia, iniciativa e responsabilidade. Externamente às organizações produtivas, a competência cumpriria o papel de ordenar as relações sociais de trabalho, no sentido de gerir as condutas e reconfigurar valores ético-políticos dos trabalhadores no processo permanente de adaptação à instabilidade social, garantindo a sua empregabilidade.

As mudanças na gestão da produção dão destaque à comunicação e supõem o deslocamento da produção de mercadoria à produção de serviço. Novos princípios de contratação e de gestão incluem os procedimentos participativos, os contratos por objetivos, as formas de avaliação e de auto-avaliação, o deslocamento do controle direto sobre as tarefas para o controle da qualidade dos produtos.

A qualificação como conceito organizador das relações de trabalho e de formação no processo de reestruturação produtiva dá lugar à noção de competência. Neste processo, o indivíduo evolui de uma lógica de ter (ter uma qualificação, ter conhecimentos), a uma lógica de ser (ser competente, ser qualificado). A competência profissional, portanto, responde à necessidade de gerar referenciais para ordenar e criar uma oferta formativa de qualidade que permita elevar o nível de qualificação dos trabalhadores de um determinado setor de atividade. Isto depende da construção de mecanismos de diálogo que aproximem os sujeitos sociais do mundo produtivo e que permitam a construção destes referenciais, a partir dos quais pode-se redesenhar e atualizar os processos formativos ${ }^{(12)}$.

A questão das competências e da relação conhecimentos-competências constante das reformas curriculares, está consubstanciada na Educação Baseada em Competências. Esta é uma forma de educação que propõe o uso de um currículo derivado de uma análise de um perfil atual ou futuro e que procura certificar-se dos progressos dos estudantes baseado no desempenho demonstrado em alguns aspectos daquele perfil ${ }^{(13)}$.

A estruturação das respostas educacionais, com base nas competências exige as seguintes ações em forma permanente ${ }^{(7)}$ :

Identificação das competências necessárias de sistemas, instituições e profissionais;

Revisão e análise das áreas críticas do desempenho de instituições e trabalhadores (habilidades, atitudes e conhecimentos) e do conhecimento (teórico e metodológico) disponível;

Ajuste ou formulação de respostas educacionais (temas, métodos e modalidades idôneos).

As vantagens de definir, claramente, as competências esperadas do profissional podem ser sintetizadas como ${ }^{(14)}$ : a oportunidade para o profissional refletir a natureza do seu trabalho dentro de uma estrutura mais ampla do que tem sido feito; estabelecer publicamente o que os membros de uma profissão estão habilitados a fazer e o que o público pode, razoavelmente, esperar deles; providenciar metas mais claras do que, normalmente, existem para as instituições de ensino e para os programas de educação permanente; tornar mais claro para o estudante o que será esperado alcançar como um recém formado, ou como alguém buscando uma especialidade, ou um status avançado.

A definição ou identificação das competências requeridas de instituições e trabalhadores deveria ser o resultado da identificação, tanto de necessidades ou demandas sociais, como de vazios ou limitações da resposta social correspondente ${ }^{(7)}$. A partir deste entendimento, competências têm sido propostas para os profissionais de saúde. A fim de compreender como estas competências estão sendo construídas na saúde pública, tendo em vista as transformações ocorridas nesta área, apresentamos a seguir o referencial das funções essenciais de saúde pública. 


\section{As transformações ocorridas na saúde pública e o referencial das Funções Essenciais de Saúde Pública}

As contribuições da saúde pública como campo teórico e de prática são mais que evidentes nos processos de reforma, seja na identificação dos determinantes da saúde sobre os quais um sistema de saúde reformado deve atuar, seja na formulação de políticas de saúde e intersetoriais ou na avaliação dos efeitos das mudanças introduzidas nas organizações e nos modelos de atenção, financiamento, regulação e prestação(15).

Estas reformas têm que dar resposta a perfis de saúde mais e mais complexos, que exigem estratégias de intervenção audazes, dirigidas a modificar as condições de vida, as iniqüidades e os comportamentos individuais e coletivos ${ }^{(2)}$. A saúde pública convencional já não é suficiente para enfrentar os desafios antes indicados, pois

[...] a saúde pública não é uma "ciência" nem uma disciplina. Os conhecimentos que requer para sustentar sua prática são previstos por diversas disciplinas articuladas em função de seu objeto. Esta articulação do conhecimento em função de seu uso (prática), em uma dimensão interdisciplinar, é a essência epistemológica da saúde pública, que pode transcender, às vezes, as disciplinas que Ihe servem, mas sem constituir uma disciplina específica ${ }^{(16-2)}$.

Nas últimas décadas o conceito de saúde pública evoluiu em várias dimensões, como dever do estado, como campo de profissionalização e de conhecimento e, sobretudo, mais recentemente, como compromisso da sociedade com seus ideais de saúde ${ }^{(15)}$. A nova visão da saúde pública implica um enfoque ampliado do conceito de políticas de saúde e de seus atores sociais relevantes. Esta elaboração se baseia no reconhecimento de práticas sociais geradoras de saúde e de sua expressão setorial em funções essenciais de saúde pública.

A saúde pública, no referencial das FESP, encontra-se em primeiro plano para análise das transformações do setor. O termo 'funções essenciais de saúde pública' entrou para o vocabulário, através do relatório de um projeto do Instituto de Medicina da Academia de Ciências dos Estados Unidos, realizado em 1988, que resultou na indicação de três funções centrais: análise/avaliação de situações-problema, desenvolvimento de políticas e garantia de provisão de serviços necessários para alcançar objetivos acordados (fossem de assistência/previdência ou promoção) ${ }^{(2)}$.

As FESP foram definidas como as condições que permitem um melhor desempenho da prática da saúde pública. Se as funções estão bem definidas e incluem todas as capacidades requeridas para uma boa prática, ficará assegurado o bom funcionamento em cada um dos campos de ação, ou esferas de trabalho da saúde pública(17).

A iniciativa "Saúde Pública nas Américas", elaborada pela Organização Panamericana da Saúde (OPAS) apresenta a definição e medição das FESP como base para melhorar a prática da saúde pública e fortalecer a liderança da autoridade sanitária em todos os níveis do Estado. Ela é coordenada pela Divisão de Desenvolvimento de Sistemas e Serviços de Saúde e inclui todas as unidades técnicas da Sede e as Representações da OPS/OMS. A Organização criou um instrumento para a medição do desempenho das FESP, conjuntamente com os Centros para o Controle e Prevenção de Enfermidades dos Estados Unidos (CDC) e o Centro Latinoamericano de Investigação em Sistemas de Saúde (CLAISS). Além disso, a iniciativa tem se beneficiado da participação e dos comentários de uma rede internacional de instituições e experts no âmbito da saúde pública ${ }^{(17)}$.

Deste trabalho, resultou a mais recente relação de funções essenciais ${ }^{(18)}$.

1. Monitoramento, avaliação e análise da situação de saúde da população;
2. Vigilância, investigação e controle de riscos e danos em saúde pública;

3. Promoção da saúde;

4. Participação dos cidadãos em saúde;

5. Desenvolvimento de políticas e capacidade institucional de planificação e gestão em saúde pública;

6. Fortalecimento da capacidade institucional de relação e fiscalização em saúde pública;

7. Avaliação e promoção do acesso eqüitativo da população a serviços de saúde necessários;

8. Desenvolvimento de recursos humanos e capacitação em saúde pública;

9. Garantia e melhoria de qualidade dos serviços de saúde individuais e coletivos;

10. Investigação em saúde pública;

11. Redução do impacto de emergências e desastres em saúde.

Nesta versão a promoção da saúde, sua base paradigmática e suas propostas estratégicas passaram a ocupar uma posição central na proposta das FESP. Por isto, considera-se $^{(19)}$ que a proposta da Organização avançou em relação às anteriores, visto que considera valores e dimensões políticas e sociais que as propostas iniciais não consideraram.

A noção de competência tem sido aplicada, no referencial das Funções Essenciais de Saúde Pública $^{(7)}$ a instituições e indivíduos. A partir deste entendimento, competências têm sido propostas para os profissionais de saúde. Elas serão apresentadas a seguir, juntamente com aquelas que têm sido propostas e discutidas na enfermagem.

\section{Competências na enfermagem e na saúde pública}

As origens da educação e do treinamento baseados na competência estão no movimento americano dos anos 60, da pedagogia baseada no desempenho. Definições de competência têm sido desenvolvidas na medicina desde os anos 70. Na enfermagem, a Conferência Australiana de Autoridades em Enfermagem começou em 1986 a desenvolver competências para o registro e inscrição de enfermeiras (20).

No Brasil, a construção de projetos político-pedagógicos que possibilitem a formação de profissionais capazes de buscar, constantemente, o conhecimento para solucionar os problemas que se apresentam das mais diversas formas e com habilidade de apropriar-se das informações e transformálas em conhecimento, num processo crítico e reflexivo, tendo compromisso com a transformação social, iniciou-se no período de 1986/89. Neste processo, enquanto tarefa coletiva dos trabalhadores de enfermagem, houve o debate ampliado e democrático em vários eventos, entre esses os Seminários Nacionais de Diretrizes para a Educação em Enfermagem no Brasil - SENADEN, quando foram discutidas e elaboradas as diretrizes curriculares para a formação dos profissionais de Enfermagem, nos diversos níveis, à luz da nova Lei de Diretrizes e Bases $^{(21)}$. A resolução CNE/CES n 3/2001 que trata das Diretrizes Curriculares Nacionais de Enfermagem estabeleceu competências gerais e específicas tendo em vista a formação de enfermeiros ${ }^{(22)}$.

Desde então, muitos estudos têm sido realizados nos Estados Unidos, na Inglaterra e na Austrália para identificação das competências na área da saúde. Estes incluem contribuições para o estudo das competências requeridas por médicos e enfermeiros para o trabalho na atenção primária e

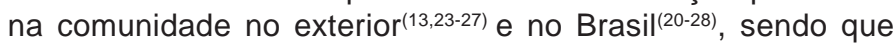
estes últimos tratam somente de competências gerais.

O referencial das funções essenciais de saúde pública deu origem a algumas propostas de competências para os profissionais de saúde. As funções centrais (análise/avaliação de situações-problema, desenvolvimento de políticas e garantia 
de provisão de serviços necessários para alcançar objetivos) fazem parte de um marco referencial, que tem influenciado um número de esforços subseqüentes nos Estados Unidos. Entre eles está a Abrangência e Padrões da Prática da Enfermagem em Saúde Pública, um enunciado emitido por organizações de enfermagem para descrever a enfermagem em saúde pública ${ }^{(29)}$.

A OPAS tem como uma de suas principais linhas de ação a definição das competências a serem incorporadas no marco estrutural das respostas educacionais utilizando as tendências sociais e de saúde da região, assim como as iniciativas e posições prévias sobre competências e prática de saúde pública. Com este entendimento, foram propostas as seguintes competências genéricas para a planificação das respostas educacionais para profissionais de saúde pública(7) : analíticas, culturais, educacionais, de comunicação, em gestão da informação, em ciências básicas da saúde, utilização de novos tipos de enfoques para o trabalho em equipe, realização de auto-aprendizagem permanente, flexibilidade de adaptação às novas modalidades de organização e de condições de trabalho, predisposição para incorporar-se às inovações e avaliar seu desempenho de forma contínua.

Estes elementos essenciais da profissão de saúde pública e a exploração da disposição e habilidades que os profissionais de saúde devem possuir para responder melhor às necessidades do público, constituem-se como componentes críticos das novas competências nucleares ou centrais comuns requeridas para o atendimento das Funções Essenciais de Saúde Pública(7). Neste contexto as competências específicas propostas foram:

1. Análise e monitoria da situação de saúde;

2. Vigilância, prevenção e controle de enfermidades transmissíveis e não transmissíveis;

3. Promoção da saúde;

4. Participação e "empowerment" social;

5. Desenvolvimento de políticas e planificação de programas;

6. Legislação e regulação em saúde pública;

7. Avaliação e promoção da eqüidade e qualidade dos serviços de saúde pessoais e baseados na comunidade;

8. Gestão de recursos humanos.;

9. Serviços específicos de saúde pública e de atenção de saúde pessoal para populações vulneráveis e de alto risco;

10.Gerência em saúde pública; planejamento e gestão financeira;

11. Saúde pública ambiental.

Esta relação de competências específicas aproxima-se muito da relação de funções essenciais proposta pela OPAS. Fazendo-se uma comparação entre estas competências específicas $^{(7)}$ e as Funções Essenciais de Saúde Pública ${ }^{(18)}$ observa-se que, para algumas, o enunciado é, inclusive, o mesmo. Outras, diferem entre si, devido ao caráter coordenador das FESP, a partir do fortalecimento e do desenvolvimento da capacidade institucional. Da mesma forma, ao desenvolvimento e a capacitação de recursos humanos garantidos pelo Estado, poderia corresponder à competência de gestão dos mesmos. A função investigação não encontra um correspondente semelhante nas competências, porém poderíamos relacionar à gerência em saúde pública, ao planejamento e à gestão financeira, por considerarmos que é imprescindível às mesmas. Nesta relação entre funções e competências se dá uma interrelação em que, não somente as competências dos profissionais de saúde são necessárias para o desenvolvimento das FESP, como, também estas exercem um papel ativo, fornecendo elementos para o desempenho das competências profissionais.

As competências das instituições e da força de trabalho têm sido indicadas como um elemento facilitador à planificação das respostas educacionais, no sentido que permite dar mais importância à prática de saúde pública nas respostas sociais, necessidades e demandas que têm sido crescentes nos últimos quinze anos. Nesta lógica, a estruturação das respostas educacionais, com base em competências, poderia ser um dos possíveis processos dinamizadores da transformação requerida pelas instituições de saúde. A definição ou identificação das competências requeridas de instituições e trabalhadores deveria ser o resultado da identificação, tanto de necessidades ou demandas sociais, como de vazios ou limitações da resposta social correspondente. Tal definição permite uma maior visibilidade das novas mudanças, existindo algumas capacidades que são mais relevantes em uma realidade determinada. As competências podem ser visualizadas em vários níveis do sistema e, em cada nível, em vários âmbitos organizacionais ou institucionais, assim como públicos ou grupos profissionais ${ }^{(7)}$.

\section{Implicações para a construção de Projetos Pedagógicos na Enfermagem}

A nova lei de Diretrizes e Bases da Educação Nacional determina que os estabelecimentos de ensino terão a incumbência de elaborar e executar sua proposta pedagógica. Esta perspectiva é visualizada a partir da construção de projetos com a participação de todos os envolvidos com o processo educativo da instituição ${ }^{(30)}$. Estes projetos são políticos porque devem assumir um compromisso com a formação do cidadão, para um tipo de sociedade e, pedagógicos, porque neles deve residir a possibilidade da efetivação da missão da instituição, que é a formação do cidadão de direitos e deveres, participativo e responsável, compromissado, crítico e criativo. Político e pedagógico têm assim uma significação indissociável.

A construção de projetos político-pedagógicos para a enfermagem, que busquem a construção deste perfil profissional, com competências, habilidades e conteúdos contemporâneos, tendo em vista a sua atuação no Sistema Único de Saúde, considerando o processo de Reforma Sanitária só será possível se houver uma discussão a respeito das transformações que estão ocorrendo no mundo do trabalho, de suas conseqüências e das possibilidades visualizadas pelos diversos atores envolvidos neste processo. Assim, como podemos suscitar esta discussão a partir das competências que estão sendo propostas para os profissionais de saúde e para a enfermagem?

A construção de competências para atender a necessidade de gerar referenciais deve ser o de gerar um debate na sociedade e, no nosso caso, na equipe de saúde e na enfermagem, sobre os ideais de saúde que se têm, os principais desafios a enfrentar, os grandes campos de atuação para alcançá-los e as responsabilidades correspondentes aos atores chaves, mais do que a elaboração de um marco normativo ou de uma lista exaustiva de atividades a cumprir desde uma lógica tecnocrática, conforme proposto pela OPAS ${ }^{(1)}$.

A noção de competência, entendida como a capacidade de articular e mobilizar conhecimentos, habilidades e atitudes, colocando-os em ação para resolver problemas e enfrentar situações de imprevisibilidade em uma dada situação concreta de trabalho e em um determinado contexto cultural, deverá levar seu todo e a cultura do local de trabalho onde se dá a ação, permitindo, ainda, incorporar a ética e os valores como elementos do desempenho competente, integrando atributos com o desempenho.

O profissional, neste contexto, deve mobilizar um saber em ato, contextualizado e construído na interação do indivíduo com a situação. O saber que deve ser mobilizado nas situações de trabalho não é de ordem, estritamente, técnica nem unidimensional, mas transversal às especialidades técnicas. $\mathrm{O}$ profissional deve ter uma visão mais global do que o especialista, colocando em prática os fragmentos emprestados a diferentes especialidades e fazendo uma combinação criteriosa desses 
fragmentos, como fruto de sua própria experiência.

Enquanto os resultados da investigação de competências expressam o que o trabalhador deve ser capaz de fazer, os currículos estabelecem as estratégias para construir essa capacidade: para resolver os problemas emergentes no exercício da função produtiva para transferir os conhecimentos, habilidades e destrezas que já possui a outros contextos profissionais. Não se trata de considerar as competências superiores e, com isto analisar as atividades desenvolvidas pelos experts com vistas a levar o conjunto de trabalhadores a um estágio equivalente de desempenho, mas de incorporar a contribuição dos trabalhadores buscando construir uma análise integrada e participativa dos processos de trabalho.

$\mathrm{Na}$ saúde pública esta é uma necessidade crescente que demanda cada vez mais a definição de perfis funcionais de pessoal que combinem, conforme Macedo "marcos conceituais e axiológicos, técnicas e destrezas provenientes de várias disciplinas"(16:7). Estes perfis devem corresponder às exigências do setor saúde que inclui as atividades de promoção, proteção, prevenção e de reabilitação, desde o autocuidado e a ajuda mútua, a atenção comunitária, os serviços profissionais de saúde até o cuidado institucional especializado e incorpora, também, o sistema de serviços de saúde, suas formas de organização e seus estilos de funcionamento ${ }^{(15)}$.

No exercício de suas atividades o profissional tem a oportunidade de reelaborar seus objetivos, sendo que os conhecimentos colocados à disposição do trabalho se modificam no contato com os problemas e acontecimentos encontrados em situações concretas, processando-se uma relação dialética entre a dimensão conceitual e a experiencial do trabalho. A esta capacidade atribui-se um exigente "uso de si por si”(31). Porém, no modelo atual, o uso de si se dá por outrem: "é o indivíduo em seu ser que é convocado"(32:3). Sua produção é o uso de si mesmo e de seus talentos como recursos de produção. No novo modo de trabalhar há uma representação ou ideal de trabalhador como se fosse possível homogeneizar aquela livre disposição de julgar/pensar/ interpretar as normas - o uso de si por si. O trabalho prescrito dá lugar a ilusão de que é possível circunscrever o sujeito através de suas prescrições ${ }^{(32)}$.

Fundamentadas sobre a valorização da implicação subjetiva no conhecimento, as competências deslocam a atenção para a atitude, o comportamento e os saberes tácitos dos trabalhadores. Atributos como capacidade de comunicação e polivalência remetem, sobretudo, à pessoa, incluindo, para além das aquisições de formação, seus atributos pessoais, as potencialidades, os desejos, os valores. A qualificação depende menos de um saber-fazer técnico do que de traços da personalidade do trabalhador ${ }^{(12)}$. Porém, ao enfatizar a dimensão da subjetividade dos trabalhadores, as competências possibilitam colocar em discussão o papel dos indivíduos na produção como sujeitos ativos, em um processo de reconstrução de sua identidade o que coloca as competências no plano da construção individual e coletiva, valorizando-se o desenvolvimento do sujeito e a formação de uma consciência coletiva e emancipatória ${ }^{(33)}$.

O processo de construção de competência, ao se basear em habilidades que envolvem todas as dimensões do indivíduo, implica rupturas na dinâmica interna dos espaços institucionais como, também, nos demais espaços que o indivíduo atua como cidadão. O trabalhador, ao articular saberes em face dos problemas encontrados em seu trabalho, atua criticamente. Ele é envolvido como um todo, rompendo-se com as delimitações impostas pelo mero fazer, atingindo-se o seu $\operatorname{ser}^{(8)}$.

O exercício da cidadania, por sua vez, alimenta-se da educação básica, sendo que o conhecimento de cada indivíduo deve ser construído ao longo de toda a sua vida a partir de quatro aprendizagens fundamentais, colocando-se ênfase no saber-ser, sobre o qual se fundariam os demais saberes: saber-fazer, saber-aprender, saber-conviver ${ }^{(34)}$. A articulação de saberes da formação geral (conhecimento científico), da formação profissional (conhecimento técnico) e as experiências de trabalho e social (qualificações tácitas), e que são mediados pela dimensão ético-política, referem-se às especificidades do trabalho em saúde ${ }^{(9)}$.

Os sistemas de competência profissional têm-se apresentado como tentativa de realizar a conciliação dos projetos dos principais sujeitos envolvidos nessa problemática como a melhor estratégia: o projeto individual, o projeto das empresas e o projeto social que tem como foco a dimensão social e coletiva do profissionalismo e o papel assumido pelo governo e pela sociedade em geral, em prover os recursos e oportunidades iguais às pessoas, para se tornarem profissionais e terem seu profissionalismo reconhecido ${ }^{(12)}$

O exercício da atividade profissional, portanto, deve evoluir do restrito plano operatório - em que o valor está na execução correta e precisa das tarefas - para um plano também reflexivo, quando se tem de enfrentar a complexidade dos processos, compreendendo-os e dominando-os. Porém, é necessário que isto se dê não apenas na dimensão micro do trabalho a fim de politizar o debate a respeito da implantação do Sistema Único de Saúde (SUS), instigando os sujeitos envolvidos a serem sujeitos reflexivos e, não, sujeitos da adesão pura e simples(35).

$\mathrm{Na}$ construção de projetos político-pedagógicos na enfermagem, portanto, é necessário que se discuta as transformações que estão ocorrendo no mundo do trabalho e no setor saúde, e que os projetos, resultado da discussão entre os diversos atores envolvidos, reflitam as necessidades da nossa clientela e as competências construídas pelos profissionais de saúde, em sua prática cotidiana. Neste processo, inserem-se as competências para o trabalho em saúde pública como forma de contribuir para as mudanças que se fazem necessárias, a fim de que se avance na implantação do SUS. Tendo em vista a contribuição da enfermagem para o trabalho em saúde pública e seu foco específico de atuação e, sendo que as enfermeiras preenchem papéis-chave em saúde pública nas Américas, cumpre que se construam modelos de formação, que viabilizem o desenvolvimento de atitudes críticas e reflexivas, em uma educação profissional específica e com instrumentos para praticar a interdisciplinaridade na saúde e elementos potencializadores da emancipação dos trabalhadores.

\section{Referências}

1. Organización Panamericana de la Salud. Funciones esenciales de salud pública: una perspectiva desde las práticas sociales: marco de referência para uma discusión regional. Washington (DC); 1998. $18 \mathrm{p}$.

2. Organización Panamericana de la Salud. División de Desarollo de Sistemas y Servicios de Salud. Funciones Esenciales de Salud Publica. Documento de posición. Washington (DC); 1997. 25 p.

3. Bodstein R. [Editorial]. Ciência e Saúde Coletiva, Rio de Janeiro 2002;7(3):398-400.

4. Ministério da Saúde (BR) In: Anais da $8^{a}$ Conferência Nacional de Saúde; 1986; Brasília (DF), Brasil. Brasília (DF): Ministério da Saúde; 1987.

5. Secretaria de Políticas de Saúde (BR). Departamento de Atenção Básica. Programa Saúde da Família [informe técnico]. Revista de Saúde Pública, São Paulo 2000 jun;34(3):316-9.

6. Maranhão EA. A construção coletiva das diretrizes curriculares nacionais dos cursos de graduação da saúde: uma contribuição para o Sistema Único de Saúde. In: Almeida M organizador. Diretrizes curriculares nacionais para os cursos universitários na área de saúde. Londrina (PR): Rede UNIDA; 2003.

7. Ruiz L. De la realidad a las disciplinas: estructuración de las respuestas educacionales com base em las competências de las instituciones y de la fuerza de trabajo. In: Organización Panamericana de la Salud. Educación en salud pública: nuevas perspectivas para las Américas. 
Washington (DC); 2001. p. 134-61.

8. Desaulniers JBR. Formação, competência e cidadania. Educação \& Sociedade, Campinas (SP) 1997 dez;18(60):51-63.

9. Deluiz N. Qualificação, competências e certificação: visão do mundo do trabalho. Formação, Brasília (DF) 2001 maio;1(2):5-15.

10. Minayo MCS. O desafio do conhecimento: pesquisa qualitativa em saúde. 6ª ed. São Paulo: HUCITEC; 1999. 269 p. (Saúde em debate; 46).

11. Antunes R. Adeus ao trabalho? $7^{\mathrm{a}}$ ed. Campinas (SP): Cortez; 2000. $199 \mathrm{p}$.

12. Ramos MN. A pedagogia das competências: autonomia ou adaptação? São Paulo: Cortez; 2001. 320 p.

13. Lane DS, Ross V. The importance of defining Physicians'competencies: lessons from preventive medicine. Academic Medicine, Washington (DC) 1994 Dec;69(12):972-4.

14. Gonczi A. Competency based assessment in the professions in Austrália. Assessment in Education; 1994;1(1)27-43.

15. Organización Panamericana de la Salud. Desafios para la educación en salud pública: la reforma sectorial y las funciones esenciales de salud pública. Washington (DC); 2000.

16. Macedo CG. La salud pública en las Américas: documento conceptual y operacional. In: Organización Panamericana de la Salud. Educación en salud pública: nuevas perspectivas para las Américas. Washington (DC); 2001. p. 1-16.

17. López AD. Iniciativa "La salud pública en las Américas": una manera de mejorar la práctica de salud pública em el continente americano. In: Organización Panamericana de la Salud. Educación en salud pública: nuevas perspectivas para las Américas. Washington (DC); 2001. p. 17-23.

18. Organización Panamericana de la Salud. Funciones esenciales de salud pública. Washington (DC): OMS; 2000. 105 p.

19. Kickbbusch I. Reflexiones sobre la salud pública em las Américas. In: Organización Panamericana de la Salud. Educación en salud pública: nuevas perspectivas para las Américas. Washington (DC); 2001. p. 24-30.

20. Silva RF. Generic competencies required by nurses and doctors in a primary health care team [dissertation of Masters Degree in Medical Education]. Scotland: University of Dundee; 1998. 117 p.

21. Ministério da Educação (BR). Diretrizes e bases da educação nacional, lei 9.394 de 20 de dezembro de 1996 e atos complementares. Diário Oficial da União, Brasília (DF) 1994 dez 16;Seção 1:19.31-302.

22. Ministério da Educação (BR). Diretrizes curriculares nacionais dos cursos de graduação em enfermagem. Diário Oficial da União, Brasília (DF) 2001 out 3;Seção 1E:131.

23. Kenyon V. Clinical competencies for community health nursing. Public
Health Nursing, Cambridge (MA) 1990;7(1):33-9.

24 . Nickel J. Community nursing competencies: a comparison of educator, administrator, and student perspectives. Public Health Nursing, Cambridge (MA) 1995;12(1)3-8.

25. Gebbie KM, Hwang I. Preparing currently employed public health nurses for changes in the health system. American Journal of Public Health, Washington (DC) 2000 May;90(5):716-21.

26. Diekemper M, Smithbattle RN, Drake MA. Bringing the population into focus: a natural development of community health nursing practice: part I. Public Health Nursing, Cambridge (MA) 1999 Feb;16(1):3-10.

27 Diekemper M, Smithbattle RN. Drake MA. Bringing the population into focus: a natural development of community health nursing practice: part II. Public Health Nursing, Cambridge (MA) 1999 Feb;16(1):3-10.

28. Silva RF, Tanaka OY. Técnica Delphi: Identificando as competências gerais do médico e do enfermeiro que atuam em atenção primária de saúde. Revista da Escola de Enfermagem da USP, São Paulo 1999 set;33(3)207-16.

29. Organización Panamericana de la Salud. La enfermería de salud pública y las funciones esenciales de salud publica. Washington (DC); 2002.

30. Saupé R, Alves ED. Contribuição à construção de projetos políticospedagógicos na enfermagem. Revista Latino-americana de. Enfermagem, Ribeirão Preto (SP) 2000 abr;8(2):60-7.

31. Schwartz Y. Os ingredientes da competência: um exercício necessário para uma questão insolúvel. Educação \& Sociedade, Campinas (SP) 1998 dez;19(65):101-40.

32. Rosa MI. Trabalho: nova modalidade de uso de si e educação: debates/ confrontos de valores. Campinas (SP): Pro-posições, 2000.

33. Deluiz N. Formação do trabalhador: produtividade e cidadania. Rio de Janeiro, Shape; 1995. 212 p.

34. Delors J. Educação: um tesouro a descobrir: relatório para a UNESCO da Comissão Internacional sobre educação para o século XXI. $6^{a}$ ed. São Paulo: Cortez; 2001. 288 p.

35. Merhy EE. E daí surge o PSF como uma continuidade e um aperfeiçoameto do PACS. Interface: Comunicação, Saúde, Educação, Botucatu (SP) 2001 ago;9:147-9.

Data de recebimento: $31 / 08 / 2003$

Data de aprovação: 30/10/2003 